\title{
HACIA UN SISTEMA DE INDICADORES IDE VULNERABILIDAD URBANA
}

\section{Josep Sorribes Salvador Perelló*}

Desde la segunda mitad del XIX hasta nuestros días, los sistemas de ciudades han conformado la matriz económica, política y social del mundo. A lo largo de más de un siglo y medio, el crecimiento económico e industrial de las naciones, se ha articulado en torno al fenómeno urbano, siendo en las ciudades donde se siguen generando las sinergias más productivas, creativas e innovadoras.

Sin embargo, los núcleos urbanos se han convertido también en un caldo de cultivo perfecto para la pobreza, la desigualdad, la marginación y la discriminación social, así como para la violencia, la contaminación y la congestión.

Desde una perspectiva disciplinar los términos anglosajones urban blight y urban renewal están íntimamente relacionados, consecutivamente, con los procesos degenerativos que se producen en determinadas áreas urbanas y con la "terapia" (pública, privada o mixta) mediante la que se intenta abortar estos procesos degenerativos.

Un área degradada (sea un barrio periférico, un polígono oficial de viviendas sociales o las áreas no renovadas de los centros históricos) adquiere tal condición cuando concurren en ella niveles de degradación física y funcional que la privan de atractivo locacional para usos residenciales o terciarios. Este atractivo locacional no es independiente, obviamente, del (que puedan ser considerados aceptables por él) sistema de valores que comparte una sociedad en un momento histórico determinado.

* Josep Sorribes es Profesor de la Universidad de Valencia. Salvador Perelló es Profesor de la Universidad Rey Juan Carlos y miembro de la Asociación Castellano-Manchega de Sociología. 


\section{LAS CAUSAS DE LA DEGRADACIÓN Y LOS REQUISITOS DE LA INTERVENCIÓN PÚBLICA}

A la degradación urbana puede llegarse por diferentes caminos: la insuficiente inversión en urbanización y equipamientos, los déficits de accesibilidad, la baja calidad constructiva, la deficiente planificación urbanística, el abandono a la que la someten los propietarios a fin de provocar una aceleración del proceso y poder sustituir los tejidos degradados por otros de mayor rentabilidad...

Sea cual fuere la variable o combinación de variables que explica el inicio del proceso de degradación, una vez iniciado éste se ponen en funcionamiento mecanismos de causación circular acumulativa que tienden a empeorar progresivamente la situación. Como ya hemos indicado la degradación supone una pérdida del atractivo locacional lo cual conlleva el abandono del área por parte tanto de la población originariamente residente como de la actividad productiva (industrial, artesanal o terciaria) para la que el entorno se convierte en un factor negativo.

Esta "huida" de las zonas degradadas no conduce, sin embargo, a un puro y simple "vaciado" del área, ya que previamente entra en acción lo que se conoce como "proceso de filtrado". Al menos parcialmente, la población que abandona la zona huyendo de la degradación, es reemplazada por grupos sociales de menor nivel de renta, forzados a reutilizar un patrimonio inmobiliario degradado y, por consiguiente, de menor coste. Pero éstos nuevos ocupantes aceleran la degradación del espacio tanto por la imposibilidad de realizar cualquier inversión de mantenimiento como por el efecto de "disuasión" que ejercen sobre el asentamiento en el área de población de rentas superiores. Los bajos niveles de renta (o la miseria) siempre van acompañados de un incremento en la inseguridad ciudadana y de la proliferación de actividades "informales" o incluso delictivas. Por tanto, la zona cada vez se degrada más y es menos atractiva.

El filtrado residencial viene acompañado también de una cierta sustitución de los usos productivos. Es habitual que los espacios abandonados por comercios, artesanía, industria o actividades terciarias "normales", sean ocupados por lo que podría denominarse "terciario degradante". Este adjetivo no debe entenderse como un juicio de valor ético, sino como una simple constatación de que la proliferación de "pubs" (con el problema adicional de la contaminación acústica y de la imposibilidad de descansar en horas nocturnas) o la extensión de la prostitución, son fenómenos que hacen cada vez más inviable que el área sea atractiva para cualquier uso urbano normalizado. Descenso de la población, degradación física de los edificios (con proliferación de espacios abandonados) y degradación funcional de los usos son las características que, en diferentes grados, podemos encontrar en las áreas degradadas de cualquier ciudad.

Si en el área degradada predomina la propiedad vertical (cosa bastante frecuente en los centros históricos) suele producirse otro círculo vicioso añadido 
que en la literatura económica se conoce como "el dilema del prisionero" y que es una de las aplicaciones más conocidas de la Teoría de Juegos. Dicho dilema es muy sencillo de comprender. Supongamos que en un área degradada, un propietario se plantea realizar una inversión de mantenimiento o mejora de su propiedad. Antes de tomar la decisión pensará, si actúa con lógica, que su inversión o bien revalorizará las propiedades colindantes o bien no será capaz por sí sola de variar la tendencia general con lo cual la rentabilidad que podría obtener de la inversión disminuye sensiblemente o desaparece. Ninguna de ambas cosas sucedería si una masa crítica mínima de propietarios invirtiera a la vez en los edificios de la zona provocando sinergias positivas y un cambio de tendencia. Pero el acuerdo entre propietarios es complejo y si todos razonan basándose en la lógica individual, el resultado es que nadie invierte y la degradación continua.

Desde una perspectiva neoliberal, no está justificado que los poderes públicos intervengan en las áreas degradadas en tanto en cuanto la degradación irá reduciendo el valor residual de las propiedades hasta que sea el propio mercado el que resuelva el problema provocando un proceso de renovación urbana. La contestación a éste argumento es bastante simple: la degradación de áreas urbanas tiene unos elevados costes sociales tanto para los residentes como para el conjunto de la ciudad y esperar que el mercado actúe puede suponer el enquistamiento del problema, sobre todo si hay, como es habitual, mejores oportunidades para la inversión inmobiliaria. Por tanto, está justificada la intervención pública, intervención que debe tener la suficiente escala como para superar los problemas ya comentados en el dilema del prisionero y ser capaz de modificar la tendencia.

Existe ciertamente un requisito que puede ser útil tener en cuenta en nuestro análisis, y es el derivado de lo que en economía se conoce como el teorema de Kaldor-Hicks que viene a resolver el callejón sin salida que suponía el óptimo paretiano según el cual no está justificada ninguna actuación que empeore la utilidad de cualquiera de los individuos afectados. Por el contrario, el mencionado teorema de Kaldor-Hicks establece que una actuación o intervención pública es aceptable siempre y cuando el incremento de los beneficios que obtienen parte de los afectados sea superior a los costes que se generan para el resto, de forma que pueda producirse la "compensación". El teorema no supone que dicha compensación se de en la práctica, sino que sea posible su realización sin que el saldo beneficios / costes desaparezca. Sin pretender que la intervención pública sólo deba producirse cuando se dan estas condiciones, pensamos que es útil razonar en términos parecidos o semejantes a fin de priorizar la intervención pública.

\section{LA RENOVACIÓN URBANA}

Ya hemos desgranado, aunque sea con brevedad, los mecanismos que están en el origen de la degradación o urban blight. La renovación urbana (o urban 
renewal) no es más que el proceso por el cual las áreas degradadas son sustituidas por áreas renovadas. La degradación y la renovación son dos procesos simultáneos, casi biológicos, que tienen en su origen variables fundamentalmente económicas aunque las consecuencias de uno y otro proceso afecten a variables sociológicas, urbanísticas y arquitectónicas.

En nuestro contexto no nos interesa analizar lo que se conoce como renovación puntual, proceso que supone simplemente la sustitución de un edificio aislado (cualquiera que sea su uso) por otro de usos más "cualificados" debiendo entenderse aquí el término "cualificado" como "más rentable". En pura lógica económica y de no mediar otras consideraciones, la operación de renovación puntual puede suponer o no la demolición del edificio preexistente a fin de incrementar el aprovechamiento del suelo vacante. Aquí lo importante es que se produzca un cambio o intensificación del uso (de viviendas populares de baja densidad a edificios de varias plantas, de zona industrial a usos residenciales o terciarios, de uso residencial a uso terciario etc..). Si aceptamos este convenio semántico, habrá renovación cuando haya cambio o intensificación del uso (incluyendo la sustitución de grupos sociales de baja renta por otros de renta superior) mientras que el término rehabilitación, debería limitarse, contrariamente a la costumbre, a aquellas operaciones de renovación en las que no se produce cambio ni intensificación del uso ni de los residentes.

Hemos dicho que no nos interesan las operaciones puntuales de renovación urbana (que son más frecuentes en los centros históricos - si se permite - o en las denominadas zonas de transición, zonas periféricas que al aumentar el radio de la ciudad han ganado centralidad y se han revalorizado sino las operaciones de renovación que tienen como objetivo abortar los procesos de degradación de áreas urbanas. Dado que la escala de la intervención debe ser razonablemente grande para vencer las inercias y dar señales claras al mercado, es bastante frecuente que la intervención sea pública o mixta y es frecuente que sea una actuación pública de envergadura (la construcción de un gran equipamiento, la apertura de una vía, la mejora de la accesibilidad proporcionada por una nueva línea de tranvía o metro...) la que actúe como detonante del proceso.

Es el mercado del suelo y los precios potenciales del mismo los que actúan como mecanismo de información y actuación. Sin un área previamente degradada se "renueva" o existen expectativas razonables de que lo haga a corto plazo, los precios del suelo reflejan el valor futuro del área, seleccionan los usos y aceleran el proceso. Caso de producirse con éxito la renovación de un área degradada, es frecuente que se de un proceso de gentrificacion (la vuelta de grupos sociales de renta alta que se habían desplazado previamente a las áreas residenciales de la corona metropolitana) pero lo que es seguro es que se incurrirá en costes sociales que suelen venir asociados a la expulsión de las áreas renovadas de la población de bajo nivel de renta o marginal. 
Pueden existir otros costes (de destrucción del patrimonio histórico artístico o de degradación medioambiental) que pueden ser mitigados y parcialmente evitados siempre que se realice con carácter previo algún tipo de análisis coste-beneficio. En este sentido, es importante subrayar que la promoción pública de viviendas sociales (de nueva planta o rehabilitadas) localizadas no de forma conjunta (a fin de evitar los conocidos "ghettos") sino distribuidas en el nuevo tejido urbano es una política sin la cual cualquier operación de renovación urbana genera costes sociales bastante más altos que los deseables y/o políticamente aceptables.

Por último, insistir en que la renovación urbana suele exigir intervenciones drásticas y de bastante escala, único modo de generar nuevas centralidades y reinvertir las tendencias de la degradación en áreas urbanas relativamente extensas (barrios o sub- barrios ).

\section{LOS NIVELES DE VULNERABILIDAD}

En el continuo que va desde la plena integración y funcionalidad urbana hasta la marginación, existen grados que no siempre se manifiestan de manera evidente. Es fácil reconocer un núcleo de población sometido a procesos de marginación dado que la "sintomatología" resulta evidente y visible. En este caso, la aplicación de un sistema de indicadores o de un método de evaluación no hace sino confirmar lo que ya es sabido.

Sin embargo, la asignatura pendiente de las estrategias de intervención públicas en el ámbito de la política urbana reside, hasta la fecha, en su escasa capacidad de anticipación a las problemáticas emergentes y su baja elasticidad ante los síntomas iniciales de vulnerabilidad. Nos encontramos ante una miopía que genera grandes efectos perversos, ya que impide estar en condiciones de identificar anticipadamente el riesgo y la tendencia a la vulnerabilidad, lastrando los posibles planes de acción preventivos. Pensemos que dentro del agregado vulnerabilidad hay una larga lista de patologías potenciales que en la mayoría de ocasiones culmina en procesos graves de degradación urbanística, principalmente porque no se han atajado a tiempo los primeros síntomas manifestados.

La cuestión que cabe plantarse a partir de este punto es cómo anticiparse al fenómeno de degradación urbana; en definitiva, cómo identificar las zonas o barrios de nuestras ciudades que presentan síntomas de vulnerabilidad.

La estrategia metodológica que proponemos para compensar la ya tradicional miopía de las políticas de intervención públicas en el ámbito urbanístico, se asienta en lo que hemos llamado Sistema de Indicadores de Vulnerabilidad Urbana (SIVU). Se trata de la articulación coherente y consistente de un conjunto de indicadores de "alerta" capaz de identificar síntomas claros de vulnerabilidad urbana, con el fin de priorizar las actuaciones que, desde una perspectiva global e 
integradora, puedan superar los procesos de degradación antes de que estos sean ya demasiado avanzados y de difícil reversión.

Este Sistema de Indicadores de Vulnerabilidad Urbana (SIVU) debería proporcionar información rigurosa y sistemática para el análisis institucional y académico de la realidad económica, social y urbanística de las áreas sometidas a la aplicación de la herramienta.

\section{METODOLOGÍA DEL S.I.V.U.}

La construcción de un Sistema de Indicadores de Vulnerabilidad Urbana (SIVU) seguiría, como cualquier otro sistema de indicadores, el siguiente proceso:

1. Elaboración del marco teórico-conceptual que integre todos los aspectos que conforman el concepto de vulnerabilidad urbana.

2. Análisis comparativo de los sistemas de referencia.

3. Determinación y análisis de las áreas de aplicación.

4. Identificación y definición de las dimensiones a analizar.

5. Selección y definición de los indicadores (tipos, función, operativización, etc.)

Tradicionalmente, los indicadores se han conformado como una herramienta de enorme capacidad para, de una forma sistemática, analizar los problemas y los impactos de determinadas políticas. Se trata de una medida que resume información sobre una cuestión en particular. Muestran tendencias y proveen de información tanto cuantitativa como cualitativa. Además, pueden convertirse en algo más que simples piezas informativas, si están diseñados como respuesta a objetivos políticos bien definidos. Los indicadores no son una simple sucesión de datos estadísticos. Lo realmente trascendente de la elaboración de indicadores, es que estén integrados en un programa o estrategia de actuación política, y que, de forma sistemática y regular, vayan reflejando tanto las necesidades, como los grados de eficiencia de las políticas puestas en práctica.

Con todo, el primer objetivo del SIVU no es evaluar la ejecución de determinadas políticas públicas en el ámbito urbano, a pesar de que su uso sistemático pueda incorporar de forma asociada esta dimensión ex post. La vocación de un SIVU es orientar las políticas de intervención, y guiarlas hacia las patologías urbanas con síntomas más evidentes con el fin de anticiparse a los procesos de degradación consolidados.

Para elaborar el SIVU es necesario considerar, como se ha señalado anteriormente, las diferentes dimensiones que son determinantes en la identificación de un área urbana vulnerable, y a las que estarán asociadas tanto las subdimensiones y variables específicas como los indicadores concretos.

Estas dimensiones, la económica, la social y la urbanística, conformarán la estructura básica del SIVU que culminará en un sistema de prevención que opere 
como una señal de "alerta" ante la aparición de síntomas de degradación y proponga qué tipo de políticas globales e integradas pueden adoptarse por la administración autonómica o local para evitar la degradación progresiva, o salir de ella, en le caso de que ya exista dicha degradación.

Aunque en el lenguaje propio de los indicadores sociales, términos como dimensión/subdimensión o área/subárea están plenamente vigentes y son de enorme utilidad a la hora de organizar y articular los sistemas de indicadores, en el ámbito de lo urbano proponemos hacer compatibles estas referencias con las de sistema/ subsistema. Esta propuesta no es gratuita, ya que el concepto de vulnerabilidad urbana que promovemos parte de un concepto de ciudad camagniano, donde para estudiar cualquier fenómeno urbano, es necesario considerar en conjunto los diversos sistemas que componen la ciudad (el sistema económico, el sistema social, el sistema físico -built and cultural heritage- y el sistema ambiental).

Los síntomas de vulnerabilidad urbana y los procesos posteriores de degradación, son consecuencia de la falta de integración equilibrada entre cada uno de estos subsistemas que son los que integran el constructo urbano.

En la estructura del sistema de indicadores que proponemos, hemos procedido a fundir el subsistema físico y el medioambiental en uno que hemos llamado urbanístico. La razón de esta aglutinación está asociada a la condición micro de los objetos de estudio que serían sometidos a este sistema de indicadores de vulnerabilidad. Hemos considerado más operativo integrar las variables clave de subsistema físico y medioambiental en un único conjunto que permita hacer un seguimiento más eficiente de la sintomatología que presentan las áreas urbanas estudiadas.

En definitiva serán tres los subsistemas en torno a los que articulamos el Sistema de Indicadores de Vulnerabilidad Urbana: El subsistema económico, el social y el urbanístico. Dentro de cada uno de ellos, encontramos una serie de variables que determinan tanto su propia evolución, como la del resto de subsistemas. Por extensión, señalar una vez más, que esta permanente interacción entre los distintos subsistemas estudiados, se conforma como el fundamento de los procesos que determinan la degradación urbana.

\section{ESTRUCTURA DEL S.I.V.U.}

Dentro del Subsistema Económico hemos introducido las variables demográficas ya que en los procesos de degradación urbana la demografía es a la vez causa y efecto. Por ejemplo, la disminución de la población suele estar asociado a la degradación en cuanto que ésta implica el abandono de usos residenciales y productivos.

Sin embargo, otra variable presente en muchos procesos de degradación es el incremento de la población inmigrada consecuencia del proceso de filtrado. Pero el incremento de la población inmigrada, siendo como es también una causa de degradación, actúa en sentido contrario a la disminución de la población. 


\begin{tabular}{|c|c|}
\hline Dimensiones & Variables \\
\hline \multirow{2}{*}{$\begin{array}{l}\text { SUBSISTEMA } \\
\text { ECONÓMICO }\end{array}$} & $\begin{array}{l}\text { Demográficas: Evolución natural de la población, } \\
\text { Evolución población migrante, lugar nacimiento } \\
\text { años de permanencia en el barrio, Índice de } \\
\text { dependencia etc. }\end{array}$ \\
\hline & $\begin{array}{l}\text { Económicas: renta per cápita, índices de pobreza, } \\
\text { paro, relación lugar de trabajo/lugar de residencia. } \\
\text { precio del suelo etc. }\end{array}$ \\
\hline \multirow{2}{*}{$\begin{array}{l}\text { SUBSISTEMA } \\
\quad \text { SOCIAL }\end{array}$} & $\begin{array}{l}\text { Recursos: institucionales, comunitarios } \\
\text { cuasi-comunitarios. }\end{array}$ \\
\hline & $\begin{array}{l}\text { Cobertura: beneficiarios de los recursos y de los } \\
\text { programas / actuaciones llevados a cabo en el área } \\
\text { objeto de estudio etc. }\end{array}$ \\
\hline \multirow{4}{*}{$\begin{array}{l}\text { SUBSISTEMA } \\
\text { URBANÍSTICO }\end{array}$} & $\begin{array}{l}\text { Localización: grado de aislamiento, proximidad a } \\
\text { infraestructuras, proximidad a instalaciones } \\
\text { industriales agresivas etc. }\end{array}$ \\
\hline & $\begin{array}{l}\text { Equipamientos: docentes, de salud, de tiempo libre y } \\
\text { ocio, de transporte, aparcamientos, alcantarillado, } \\
\text { agua potable y riego, electricidad y alumbrado, } \\
\text { telefonía y telecomunicaciones, gas canalizado, } \\
\text { recogida de basuras, pavimentación de aceras y } \\
\text { calzadas, arbolado, jardinería, mobiliario urbano etc. }\end{array}$ \\
\hline & $\begin{array}{l}\text { Diseño Urbano: especialización funcional de los } \\
\text { edificios, especialización funcional del sistema } \\
\text { viario, variabilidad tipológica de los edificios etc. }\end{array}$ \\
\hline & $\begin{array}{l}\text { Habitabilidad: edad de la edificación, grado de } \\
\text { rehabilitación, grado de renovación, condiciones de } \\
\text { habitabilidad. }\end{array}$ \\
\hline
\end{tabular}

Otras variables demográficas a considerar son el lugar de nacimiento y, sobretodo, los años de permanencia en el barrio, en la medida en que ésta última variable está positivamente correlacionada con los niveles de identificación colectiva.

Por último lo que se conoce como índice de dependencia, o porcentaje que supone la población de más de 65 años y la de menos de 16 en relación al total, es 
otra variable de interés porque la ausencia de servicios públicos específicos que atiendan las necesidades de estos colectivos pueden derivar con facilidad en situaciones de marginalidad.

Al igual que sucede con las variables demográficas, existen algunas variables económicas básicas que es preciso considerar en ésta fase previa a la definición de indicadores y la construcción del correspondiente sistema. Sin duda, la primera variable a considerar es la renta familiar disponible. Su cuantía global y distribución nos permitirá tanto establecer la importancia objetiva de las situaciones de pobreza, como correlacionar dicha variable con el estado de conservación física de los inmuebles, la presencia de actividad económica privada (comercio y servicios) y la presencia o ausencia de conflictos sociales explícitos o implícitos. En sentido contrario actúa, lógicamente, la tasa de paro.

En otro orden de cosas, los barrios dormitorio, es decir, aquellos en los que la relación entre lugares de trabajo localizados y población ocupada residente en el barrio es muy baja, favorecen la aparición de situaciones de degradación urbana puesto que no existe en éstos casos ningún motivo de atracción para la población residente en otros barrios, atracción que sólo se produce en presencia de algún gran equipamiento, centro de comunicación o localización significativa de actividad productiva. En dicha circunstancia, el barrio en cuestión queda marginado de la dinámica urbana y la atención de los poderes públicos suele disminuir.

Los cambios en la accesibilidad es otra de las variables claves puesto que, si dichos cambios incrementan la accesibilidad, la revalorización del entorno es mucho más fácil. Por el contrario, situaciones que conduzcan a un mayor aislamiento desde el punto de vista de la accesibilidad al conjunto del sistema favorece el desarrollo de "ghettos" y de grupos marginales para los que la deficiente accesibilidad es una ventaja localizativa.

Un forma de comprobar si el estado de degradación del barrio es escaso, alto o muy alto es conocer el "quantum" de $\operatorname{los}^{2}$ que han cambiado de uso o han experimentado una intensificación del mismo, siempre y cuando, como es lógico, podamos comparar este dato con el conjunto de la ciudad o con lo sucedido en otros barrios.

Por último, los precios del suelo urbano son la variable económica que más influye en la dinámica de degradación/ renovación. Los precios del suelo, a pesar de las imperfecciones del mercado, actúan como transmisores de señales para la toma de decisiones de los agentes privados. Su disminución o estancamiento es símbolo inequívoco de que el mercado "castiga" la zona, mientras que cuando los precios potenciales superan a los reales se puede hablar de expectativas positivas y de incremento de la probabilidad de renovación. Un aumento de los precios, a la vez que actúa como variable que selecciona qué usos son posibles en la zona y cuales no, es una señal inequívoca de expectativas positivas o de cambios ya acaecidos que revalorizan el entorno. 
El subsistema social, por su enorme amplitud y especiales características, presenta una gran complejidad a la hora de articular en torno a él una matriz de indicadores que además se integre de forma estable en el SIVU

Por esta razón, y con el fin de ganar en operatividad analítica, el subsistema social se ha desagregado en dos dimensiones básicas de análisis: la de recursos y la de cobertura. Lógicamente, a cada una de estas macro dimensiones, le corresponden una serie de variables que son las que alimentan el SIVU.

La consideración del concepto de recurso social se encuentra íntimamente ligada al de necesidad. De hecho, estimamos la existencia de un recurso en la medida que se reconoce como un instrumento para la resolución de necesidades. Al situarnos en el plano de la comunidad, los recursos aparecen como el conjunto de materiales, formas organizativas, y potencialidades efectivas (habilidades, conocimientos, acceso de canales de información, etc.), que hacen viable el barrio como comunidad integrada, dotándolo de estabilidad y capacidad evolutiva.

En este sentido, el concepto recurso va inevitablemente ligado al de colectivo, ya que estos, a través de una determinada dotación de recursos sociales, estarán en mejores o peores condiciones de cubrir sus necesidades en los diferentes niveles (individual, familiar, grupal, comunitario etc.). Como puede fácilmente observarse, el concepto recurso se deriva directamente de qué se entienda por problema y en función de qué criterios se defina la necesidad.

Es posible que existan diferencias sustanciales entre los diferentes segmentos de población que configuran la comunidad, en su apreciación de las necesidades y en su disponibilidad de acceso a mecanismos e instrumentos para hacerles frente. Colectivos de acción específica dentro del campo de los servicios sociales como las familias, las mujeres, la infancia, la juventud, la tercera edad, los discapacitados, los drogodependientes, las minorías étnicas, los inmigrantes, los transeúntes etc. presentan un orden de necesidades de actuación muy diferentes, con la cautela añadida que la utilización de mecanismos o actuaciones públicas inadecuadas, podrían incluso provocar o acentuar los procesos degenerativos o los síntomas de vulnerabilidad que presenta cada barrio o comunidad.

Por todo ello, proponemos integrar en el SIVU la siguiente clasificación de recursos para su posterior traducción a indicadores operativizados concretos: recursos institucionales, comunitarios y cuasi-comunitarios.

Por otro lado, entendemos por recursos institucionales aquellos que se generan por la intervención de las instituciones públicas en su función de dotar de infraestructuras y servicios a la comunidad. Incluyen por tanto servicios del ámbito escolar, sanitario, laboral, socio-asistencial, etc.

Entre las características de este tipo de recursos cabe señalar el hecho de que se gestionan, en general, por personal vinculado directamente a la institución responsable; el acceso a los mismos se realiza en función de la existencia de situaciones previstas formalmente, es decir, su aplicación se 
produce en respuesta a que se manifiesten condiciones preestablecidas anteriormente de forma mas o menos objetiva y no a percepciones subjetivas de necesidad. El establecimiento de este tipo de recursos se fundamenta básicamente en criterios de necesidad normativos.

Los recursos comunitarios son los que emergen de la propia dinámica del barrio o sistema comunitario como respuesta a necesidades experimentadas y expresadas. Constituyen un complejo entramado de relaciones y mecanismos de resolución de necesidades que generalmente no se hace explícito en la comunidad y que sin embargo caracteriza la dinámica del sistema, condicionando sus propios parámetros y por tanto las potencialidades evolutivas de adaptación al entorno, así como la capacidad de mantenimiento de la propia autonomía.

Dentro de la categoría Recursos Cuasi-Comunitarios ubicamos los recursos que se gestionan totalmente o en parte por componentes de la propia comunidad o barrio, a partir de formas organizativas no surgidas directamente desde el sistema comunitario, sino de macro-organizaciones generalmente religiosas, sociopolíticas o como una prolongación de las instituciones públicas a través de programas de participación y/o descentralización de la gestión de servicios.

Este tipo de organizaciones habilitan generalmente recursos complementarios a los institucionales y comunitarios. Entre sus características básicas cabe considerar que suelen disponer de poder referencial y normativo, incidiendo por tanto en los valores morales, ideológicos, etc. de la comunidad, contando para ello con líderes más a menos entrenados. En la terminología tradicional correspondería a lo que se denomina organizaciones de control normativo.

Desde el punto de vista de la cobertura, debemos descender al nivel de los esfuerzos y programas/actuaciones concretos que se han llevado a cabo en cada barrio objeto de estudio. Si en la dimensión de recursos el objetivo es contar con una matriz de indicadores que podríamos calificar de inputs, ya que hacen referencia a los medios institucionales, comunitarios y cuasi-comunitarios disponibles para cubrir las necesidades de cada uno de los colectivos de referencia, en la dimensión que nos ocupa, la de cobertura, el objetivo es articular indicadores de outputs que nos permitan contrastar el grado de eficacia con que los inputs de traducen en cobertura real.

Sólo así estaremos en condiciones de identificar los ámbitos y colectivos que presentan una grado de desprotección y vulnerabilidad mayor. En sí misma, esta dimensión analítica ya conforma un prediagnóstico claro de la vulnerabilidad emergente en cada barrio, ya que la realidad del subsistema social es el reflejo de toda una serie de patologías propias de los otros dos grandes subsistemas que integran el SIVU, el económico y el urbanístico.

Dentro del subsistema urbanístico hemos destacado cuatro tipos de variables relevantes para el SIVU: de localización, de equipamientos, de habitabilidad de las viviendas. 
Dentro de las primeras, las variables de localización es importante hacer referencia al grado de aislamiento con respecto a los tejidos urbanos circundantes. Un barrio o área urbana puede estar rodeado por otros barrios, formando un continuo con ellos, o bien puede estar completamente aislado con respecto a otros tejidos urbanos (como una isla) y rodeado por un mar de carreteras y espacios vacíos sin carácter urbano. La segunda localización es desfavorable, ya que el barrio deja de ser una porción de la ciudad y pasa a percibirse (por sus habitantes y por los que no lo son) como una entidad separada, introvertida y sin más conexiones con el resto de áreas urbanas que las redes de infraestructuras de transporte.

Entre los dos extremos (barrio-isla o barrio como porción de ciudad, existen situaciones intermedias. Un barrio puede estar conectado con el resto del continuo urbano por uno de sus lados, por dos o por tres, y ello determina el carácter más o menos periférico del barrio, en función de la porción de su perímetro que tiene condición de borde urbano.

Otro aspecto relevante desde el punto de vista de la localización es la proximidad a infraestructuras de transporte agresivas (autovías o autopistas, líneas de ferrocarril, aeropuertos, etc.). La cercanía mayor o menor a este tipo de infraestructuras, que pueden generar tanto excesos de ruido como problemas de inseguridad es una variable de localización que debe tenerse en cuenta.

De la misma forma, la proximidad a instalaciones industriales agresivas y/ o antiurbanas puede producir contaminación o problemas higiénico-sanitarios en general, incluyendo aquellas instalaciones que, aun no generando contaminación, generen un ambiente visual antiurbano por la índole y extensión de las mismas (almacenamiento de contenedores, por ejemplo).

Complementariamente, si los agentes urbanos entienden que existen expectativas razonables de modificaciones drásticas en la situación de un área urbana, por determinaciones de planeamiento, se puede inducir el deterioro de la misma.

Respecto a las variables de equipamientos y servicios, la relación de ítems que se deberían incorporar al SIVU para intentar identificar los primeros síntomas de vulnerabilidad de una zona o espacio urbano concreto serían los siguientes:

a) Dotación de parques vecinales, plazas y espacios públicos, entendiendo como tales los que pueden considerarse como tales, no los espacios libres residuales o los espacios verdes intersticiales sin cualidad de parque público.

b) Dotación de equipamientos docentes (fundamentalmente escuelas como dotación propia del nivel de unidad vecinal, pero también otros edificios docentes para enseñanzas medias, profesionales o universitarias).

c) Dotación de equipamientos deportivos a escala de barrio.

d) Dotación de locales comerciales, fundamentalmante tiendas y supermercados en las plantas bajas, pero también mercados permanentes o semanales. La actividad comercial tiene un papel urbano fundamental como soporte de la vida pública y la animación de las calles. 
e) Existencia de servicios de transporte público (bus / metro / taxi) y grado de eficiencia de los mismos para una adecuada conexión del área con el centro y con otras áreas urbanas.

f) Dotación de aparcamientos para turismos, tanto para residentes como para visitantes y vehículos de servicio, en la vía pública y en los propios inmuebles.

g) Grado de calidad de la urbanización: dotación de servicios urbanos y mobiliario en las calles.

Incluyendo la valoración del estado de los elementos de urbanización siguientes:

- Alcantarillado.

- Agua potable y riego.

- Electricidad y alumbrado.

- Telefonía y telecomunicaciones.

- Gas canalizado.

- Recogida de basuras.

- Pavimentación de aceras y calzadas.

- Arbolado, jardinería y mobiliario urbano (papeleras, bancos, fuentes, etc).

La tercera de las variables que articulan en subsistema físico del SIVU es la que hace referencia al diseño urbano. Se trata de incorporar al sistema indicadores clave que permitan evaluar:

a) Grado de especialización funcional de los edificios. La monofuncionalidad (edificios sólo de viviendas) es problemática, y la mezcla de usos es deseable. Los edificios mixtos (con locales comerciales en planta baja y viviendas en las plantas superiores) son el tipo base para construir ciudad, para poder obtener tejidos residenciales capaces de alcanzar carácter urbano.

b) Grado de especialización funcional del sistema viario. La calle mixta (con aceras para peatones a ambos lados, aparcamientos y calzada para vehículos) garantiza un grado de urbanidad mayor que la calle especializada (calle peatonal o calle para el tráfico exclusivamente). La calle urbana no debe ser una carretera (sólo coches) ni un parking (sólo aparcamiento) ni un camino (sólo caminantes), sino todo a la vez.

c) Grado de especialización funcional del barrio (zonificación). La especialización funcional es también problemática a la escala del barrio: si los edificios públicos del mismo (mercado, parque, etc) están concentrados por un lado y las viviendas por otro se crean zonas sin actividad durante el día y otras sin actividad durante la noche.

d) Grado de vinculación de los edificios con las calles. En los tejidos residenciales formados por manzanas, las ventanas de las casas se abren a las calles, y desde éstas se accede a los zaguanes y a las tiendas. En los tejidos formados por bloques de edificación abierta, en cambio, los edificios son independientes de las calles, y las ventanas de las casas se abren al espacio intermedio entre bloques, que no suele ser la calle. Las variables anteriores, sumadas, hacen que la calle, 
soporte del espacio público y la vida urbana, pierda contenido como tal: las aceras, total o parcialmente, dejan de ser el recorrido de acceso a zaguanes y tiendas y se convierten en lugares menos transitados y, por ello, más inseguros.

e) Sobreabundancia de espacios libres intersticiales (sin condición urbana). La existencia de espacios libres verdes con carácter residual, como relleno de los restos entre edificios separados, en proporción excesiva, que imposibilita su mantenimiento por los municipios. La proporción entre suelo libre y suelo edificado es un índice de urbanidad.

f) Existencia de espacios libres privados asociados a las viviendas o a los edificios. El tejido esponjoso que resulta de la agregación de casas entre medianeras en un pueblo o en los tejidos urbanos históricos, con patios privados traseros, o los modelos de manzanas con espacio libre interior comunitario pero privado, con acceso controlado desde el exterior por vallas o por el propio perímetro edificado, son modelos de ocupación de suelo que, de existir, aportan a las viviendas un valor añadido.

g) Grado de variedad tipológica de los edificios. La existencia de demasiados edificios iguales en un barrio es problemática. La monotonía de los edificios (exceso de edificios iguales) genera un paisaje urbano triste y, además, suele ser indicativa de un exceso de uniformidad en el parque de viviendas, y por tanto de una dificultad del mismo para albergar grupos de habitantes (familiares o no) variados, como sería deseable.

Respecto a la variable habitabilidad de las viviendas, el SIVU se asentará en indicadores que evaluarán:

a) Edad de la edificación. La excesiva igualdad (todos los edificios nuevos o todos viejos) no es preferible a una composición variada de edificios nuevos, menos nuevos y viejos.

b) Grado de rehabilitación. La rehabilitación de fachadas es un parámetro observable a simple vista, mientras que la remodelación interior de cocinas y baños de las viviendas es imposible de medir.

c) Grado de renovación. La demolición de edificios viejos para la construcción de otros nuevos, así como la rehabilitación integral de edificios, son obras sujetas a licencia municipal que indican actividad constructora.

d) Condiciones generales de habitabilidad de viviendas. Existencia de ascensor, tipologías, tamaños, características de zaguanes y escaleras, son aspectos que pueden considerarse.

e) Percepción de la vivienda propia por el usuario. Hay tipos de edificios impopulares, que se perciben como lugares no deseables para vivir: edificios demasiado altos o monótonos en su composición de huecos (colmenas), o edificios con mala calidad en los acabados de fachadas. Una valoración estética de los edificios adoptando el punto de vista del gusto común del usuario no es desechable. 


\section{UTILIDAD DEL S.I.V.U.}

Para que un sistema de indicadores, del tipo que sea, sea realmente operativo, al margen de estar bien articulado y ponderado en sus dimensiones, debe estar insertado en una estrategia pública de actuación. En el caso de un SIVU, este planteamiento es aún más urgente, ya que el fenómeno/s que estamos intentando combatir, son de carácter muy micro, y de forma habitual, alteran la vida cotidiana de la mayoría de nuestras ciudades.

Un Sistema de Indicadores de Vulnerabilidad Urbana (SIVU) tiene como objetivo prioritario establecer una tipología de áreas o barrios vulnerables que permita implementar políticas de intervención a través de programas e intervenciones concretos. Esta estrategia integrada de actuación, debería incorporar complementariamente a los afectados/protagonistas, tanto en el proceso de aproximación a la realidad del objeto de estudio, como en la fase de actuación pública destinada a combatir los desequilibrios existentes en las áreas urbanas estudiadas.

En ese sentido, el diseño y puesta a punto de una práctica de evaluación adecuada, será la última pieza del puzzle que nos permitirá acometer con mayor rigurosidad, el reto de anticiparnos a la degradación. 


\section{BIBLIOGRAFÍA}

ALVIRA, F. (1991): "Metodología de la Evaluación de Programas", en Cuadernos metodológicos, $\mathrm{n}^{\mathrm{O}} 2$, CIS, Madrid.

BOIRA, J. V. y GAJA, F. (1994): "Planeamiento y realidad urbana en la ciudad de Valencia (1939-1989)", en Cuadernos de Geografía, no 55, pp. 63-89.

BORJA, J. (1998): Estado y ciudad, PPU, Barcelona.

CAMAGNI, R. Ed. (1996): Economia e Pianificazione della Città Sostenible, Il Mulino, Bologna.

- (1999): "El desarrollo urbano sostenible. Razones y fundamentos de un programa de investigación", en Papeles de Economía, n 80, Madrid.

CAMPOS VENUTI, G. (1996): Pensar la ciudad, vitalidad y límites del plan urbanístico, Universidad de Valladolid, Instituto de Urbanística, Valladolid.

DE ESTEBAN, A. (1981): Las áreas metropolitanas, un análisis ecológico, CIS, Madrid.

- (1982): Estudio comparado de estándares de equipamientos. 1. Teoría y análisis 2. Normativa, MOPU, Madrid, 2 vols.

-(1989): "La incidencia actual y futura del urbanismo en las actividades económicas" en VV.AA., La intervención empresarial en el urbanismo, CEIM, Madrid, pp. 16-27.

- (1990): Cuantificación de la demanda de oficinas en el sector privado, Consejo de Política Territorial, Madrid.

DE ESTEBAN, A. y ALVIRA, F. (1983): Sociología y medio ambiente, Centro de Estudios de Ordenación del Territorio y Medio Ambiente, Madrid.

DE ESTEBAN, A. y PERELLÓ, S. (2003): “Ordenación del territorio y sostenibilidad: génesis, herramientas y metodología”, en Sistema, n 174-175, pp. 215-224.

DE ESTEBAN, G. y BENAYAS, J. (1998): Problemática de las Grandes Áreas Metropolitanas, Madrid, Barcelona, Valencia y Bilbao, Actas de las IX Jornadas sobre el Paisaje, Asociación para el Estudio del Paisaje, Centro Nacional de Educación Ambiental, Segovia.

LEONARDO, J. (1989): Estructura urbana y diferenciación residencial, el caso de Bilbao, CIS, Madrid.

MATEOS, A. y MORAL, F. (2000): La Unión Europea y la inmigración extranjera desde la perspectiva de los jóvenes, CIS, Madrid.

PERELLÓ, S. (2001a): "Sobre el concepto de sostenibilidad urbana, su aplicación a las áreas metropolitanas de los países desarrollados y la utilidad de los indicadores", en SORRIBES, J. Ed., PERELLÓ, S. y IZQUIERDO, V., Op. Cit., pp. 99-117.

- (2001b): "Algunas experiencias de interés", en SORRIBES, J. Ed., PERELLÓ, S. y IZQUIERDO, V., Op. Cit., pp. 137-191. 
SETIÉN, M.L. (1993): Indicadores Sociales de Calidad de Vida. Un sistema de medición aplicado al País Vasco, CIS, Madrid.

SORRIBES, J. y PERELLÓ, S. (2001): "La ciudad en la historia", en SORRIBES, J. Ed., PERELLÓ, S. y IZQUIERDO, V., Op. Cit., pp. 19-43.

SORRIBES, J. Ed., PERELLÓ, S. y IZQUIERDO, V. (2001): Las ciudades del siglo XXI, UNED, Valencia.

UÑA JUÁREZ, O. (1979a): “Teoría del espacio geográfico al interno de las filosofías de la Historia: Hegel y Toynbee", en Sociedad y ejercicios de razón, Ediciones Escurialenses, El Escorial, pp.161-177.

-(1979b): "Las tipologías históricas de la ciudad y la sociología urbana", en Sociedad y ejercicios de razón, Ediciones Escurialenses, El Escorial, pp.195-231.

- (1979c): "La juventud y los espacios significativos de la ciudad", en Teoría Sociológica y Comunicación, Australian Hispanic Studies Society, AucklandMadrid-Melbourne, pp. 307-320.

UÑA, O. y HERNÁNDEZ, A. (2003): Diccionario de Sociología, ESIC, Madrid. VEDUNG, E. (1997): Evaluación de políticas públicas y programas, Ministerio de Trabajo y Asuntos Sociales, Instituto Nacional de Servicios Sociales, Madrid. VERGITI, S. (1989): Dimensioni sociali e territoriali della qualità della vita, Euroroma, Roma. 